\title{
LeS MASQUES FIN-DE-SIÈCLE. PoÉTIQUES DE LA SIMUlaton ET DE LA DISSIMULATION DANS LE ROMAN SYMBOLISTE
}

\author{
Eva VOLDŘICHOVÁ BERÁNKOVÁ \\ Université Charles, Prague
}

\begin{abstract}
En): Faithful to the teachings of Baudelaire, for whom "the exclusive sense of the Truth [...] oppresses and stifles Beauty" and who defines poetry as "what is completely true only in another world", the authors of the fin-de-siècle develop a real "aesthetics of elsewhere" in which strategies of simulation and dissimulation play a crucial role. Pretending to be someone else, "lying" about the genre of their own texts, multiplying misunderstandings between the characters (male or female? human being or artificial creature? alive or dead?), keeping the reader in suspense by endless digressions which dissimulate the real purpose of the story or, on the contrary, simulating a conclusion that will be disproved by the rest of the novel, Symbolist novelists multiply traps to arouse the reader to 'extreme consciousness', unthinkable without mask-wearing. Based on a corpus of texts by Remy de Gourmont, Villiers de l'Isle-Adam, Édouard Dujardin and others writers, the article summarizes different ways of simulation and dissimulation and deduces from them some general principles of Symbolist poetics.
\end{abstract}

Keywords (En): Symbolist novel ; simulation ; dissimulation ; pseudonym ; literary genres

Mots-clés (Fr) : roman symboliste ; simulation ; dissimulation ; pseudonyme ; genres littéraires

\section{Introduction}

Fidèles aux enseignements de Baudelaire, pour qui «le goût exclusif du Vrai (si noble quand il est limité à ses véritables applications) opprime [...] et étouffe le goût du Beau » (BAUDELAIRE, 1868 : 257) et qui définit l'œuvre poétique comme «ce qui n'est complètement vrai que dans un autre monde » (BAUDELAIRE, 1939 :299), les auteurs fin-de-siècle développent toute une «esthétique de l'Ailleurs » («anywhere out of the world», comme dirait Huysmans) dans le cadre de laquelle des stratégies de simulation et de dissimulation jouent un rôle crucial.

\section{Le jeu des pseudonymes}

La plupart des auteurs de l'époque «mentent »d'emblée à propos de leur propre identité, se cachant derrière des noms de plume plus ou moins fantaisistes. Des étrangers aspirent à revêtir une allure plus française. Ainsi, Kapitolina Sergueïevna Mestcherskaïa (1839-1918) devient Tola Dorian, Joannis Papadiamantopoulos (1856-1910) signe ses poèmes Jean Moréas et Wilhelm Apollinaris de Kostrowitski (1880-1918) s'illustre comme Guillaume Apollinaire.

Les Français, eux aussi, échangent volontiers des noms banals ou difficilement mémorisables contre des pseudonymes plus élégants, de sorte que Léon-Émile Petitdidier (1839-1927) se métamorphose en Émile Blémont, François Anatole Thibault (1844-1924) en Anatole France, Paul Alexandre Martin Duval (1855- 
1906) en Jean Lorrain, Séraphin Justin François Boex (1859-1848) en J.-H. Rosny jeune, Émile Albert Kayenberg (1860-1929) en Albert Giraud, Eugène Léon Édouard Torquet (1860-1918) en John-Antoine Nau, Georges Grassal de Choffat (1867-1905) en Hugues Rebell, Camille Laurent Célestin Faust (1872-1945) en Camille Mauclair, Frédéric-Charles Bargone (1876-1957) en Claude Farrère et Louis Henri Jean Farigoule (1885-1972) en Jules Romains.

Parfois, une petite touche originale suffit comme chez Mallarmé (18421898) qui anglicise son prénom Étienne en Stéphane ou chez Corbière (18451875) qui troque son Édouard-Joachim contre un Tristan plus littéraire, anticipant ainsi sur Samuel Rosenstock (1896-1963) qui deviendra, beaucoup plus tard, Tristan Tzara.

La folie des grandeurs pousse de nombreux ambitieux à se parer des titres de noblesse : Isidore Lucien Ducasse (1846-1870) s'inspire du roman Latréamont (1838) d'Eugène Sue pour se forger le pseudonyme de comte de Lautréamont. Joseph-Aimé Peladan (1858-1918) se fait appeler le Sâr («roi» en assyrien) Mérodack (un prénom babylonien) Joséphin Peladan, Paul-Pierre Roux (18611940) devient Saint-Pol-Roux et Vincent Biétrix (1878-1945) publie ses recueils de poésie sous le nom de Tancrède de Visan.

Des esprits plus ludiques cumulent plusieurs pseudonymes à l'instar de Laurent Tailhade (1854-1919) qui signe ses œuvres respectivement Azède, El Cachetero, Dom Junipérien, Lorenzaccio, Patte-Pelue, Renzi ou Tybalt. Quant à André Gide (1869-1952), il intitule son premier recueil Les Cahiers d'André Walter (1891) et, encore en 1917, il se désignera comme Fabrice dans des pages trop intimes de son propre Journal.

Des ouvrages collectifs sont souvent signés par un seul écrivain fictionnel. En 1880, Gabriel Vicaire et Henri Beauclair s'amusent ainsi à composer Les Déliquescences, poèmes décadents d'Adoré Floupette, un recueil qui parodie et popularise en même temps l'esthétique fin-de-siècle. Trois ans après, Paul Adam et Félix Fénéon publient leur fameux Glossaire pour servir à l'intelligence des auteurs décadents et symbolistes (1888) sous le pseudonyme de Jacques Plowert.

Aux écrivains femmes, notamment celles qui s'aventurent dans le genre érotique voire lesbien, les pseudonymes s'imposent obligatoirement : Jeanne Scrive (1857-1908) devient ainsi Jane de la Vaudère, Marguerite Vallette-Eymery (1860-1953) Rachilde ${ }^{1}$ et Pauline Mary Tarn (1877-1909) Renée Vivien ou bien «Sapho 1900 ». Il en va de même pour les éditrices : quand elle travaille comme secrétaire de rédaction chez Gallimard, Anne Desclos (1907-1998) voile son identité sexuelle derrière un pseudonyme plus neutre, Dominique Aury ${ }^{2}$, et Jeanne Loviton (1903-1996) succède à Robert Denoël à la direction des éditions éponymes sous le pseudonyme de Jean Voilier.

Le nom de plume peut même traduire une soumission explicite, comme dans le cas de Colette qui est forcée par son premier mari à publier Claudine à l'école (1900) sous le nom de «Willy». Dans Claudine à Paris (1901), Claudine en

\footnotetext{
${ }^{1}$ En 1884, Rachilde publie son Monsieur Vénus avec un coauteur masculin appelé Francis Talman, dont on n'a jamais pu déterminer s'il existait bel et bien, ou si elle l'avait inventé. Quoi qu'il en soit, dès la réédition française de 1889 , Talman est évincé du livre pour ne plus jamais réapparaitre.

${ }^{2}$ En 1954, elle publiera sa sulfureuse Historie d'O sous le pseudonyme Pauline Réage.
} 
ménage (1902) et Claudine s'en va (1903) les époux s'accordent sur un compromis en signant «Willy et Colette». Au fur et à mesure que l'écrivain se libère de la tutelle maritale, elle renverse l'ordre des noms, de sorte que Dialogues de bêtes (1905) seraient écrits par «Colette Willy » et le reste de sa production par la seule « Colette ».

\section{Les dissimulations génériques}

S'ils se font passer pour d'autres, les auteurs fin-de-siècle ont l'habitude de ruser également à propos du genre de leurs propres textes. Comme Jules Renard le rappelle dans son Journal, après la fin du naturalisme, « la formule nouvelle du roman, c'est de ne pas faire du roman » (RENARD, 1990:70). En effet, le genre étant trop entaché par le réalisme et le déterminisme zolien, les symbolistes l'esquivent ou, du moins, l'atténuent au moyen de préfaces, avant-propos ou soustitres programmatiques à visée didactique : Sixtine (1890) de Remy de Gourmont se trouve ainsi définie comme un « roman de la vie cérébrale ». Le Songe d'une femme (1899) est désigné comme un «roman familier », à savoir un type de texte qui fait s'enchevêtrer des éléments trop hétérogènes (bribes de correspondance, pages de journaux intimes, contes mis en abyme) pour n'être qu'un roman tout court. Le «Fantôme », toujours du même auteur, est désigné comme «conte » quand il paraît en volume, mais il est présenté aux lecteurs du Mercure de France en 1892 comme "roman », tandis qu' Une nuit au Luxembourg (1906) s'inspire du motif d'un «manuscrit trouvé », très prisé au XVIII" siècle, ce qui permet à Remy de Gourmont de faire du roman sans en avoir l'air.

Tout porte à croire que, d'une part, les symbolistes ont besoin de l'étiquette « roman » pour attirer le lecteur (la plupart des auteurs fin-de-siècle sont en réalité beaucoup moins élitistes et méprisants du grand public qu'ils ne le prétendent), mais en même temps, ils veulent se démarquer officiellement du réalisme voire naturalisme qui ont jusque-là défini ce genre. Leur volonté de vendre ne cesse ainsi de se heurter aux polémiques esthétiques. D'où leurs étranges stratégies publicitaires, comme celle que Georges Rodenbach a utilisée pour diffuser son chef-d'œuvre Bruges-la-Morte (1892). Le livre sort d'abord sous forme de dix épisodes dans Le Figaro. À la demande de l'éditeur Flammarion, il est modifié par son auteur qui y ajoute 35 photographies et deux chapitres (VI et XI), ce qui permet de publier l'ouvrage dans le cadre d'une série de «romans illustrés par la photographie » (CARAION, 2003 : 211). Ce genre, à la fois très populaire et très commercial, est pourtant régulièrement dénoncé dans le Mercure de France, organe officiel des symbolistes, qui souligne l'incompatibilité des photographies avec leur esthétique (IBELS, 1898 : 97).

En effet, si les photos sont admises comme support de la description dans les récits de voyage, les biographies et les romans réalistes qui ont pour "idéal la description exacte de la vie humaine et de l'être humain » (IBELS, 1898:109), elles ne sont pas acceptables pour évoquer les paysages d'âme que les symbolistes entendent suggérer au lecteur.

La stratégie de Rodenbach consiste donc en une double dissimulation. L'horizon d'attente du grand public est rassuré par lui en obtenant un beau livre de 
collection, à la typographie aérée, accompagné de 35 photos de la ville de Bruges et entrant parfaitement dans la catégorie bourgeoise du «roman illustré ». En même temps, à l'enquête lancée par ses collègues symbolistes du Mercure de France Rodenbach répond à la Salomon :

Certes l'idée de faire l'illustration d'un roman par la photographie est ingénieuse, mais un lecteur un peu subtil aimera toujours mieux s'imaginer par lui-même les personnages, puisqu'un livre n'est qu'un point de départ, un prétexte et un canevas à rêver. [...] Quant à moi, vous comprendrez que je m'intéresse simplement au texte. (IBELS, 1898 : 97-113).

Bref, la plupart des symbolistes seront toujours obligés de ménager la chèvre et le chou et de soigner la bonne réception de leurs livres par des bourgeois solvables, tout en clamant haut et fort leur intention de réduire le lectorat aux purs connaisseurs. Vendre un livre au grand public comme divertissant et le faire passer en même temps pour un ouvrage élitiste, hermétique, novateur voire révolutionnaire devant ses confrères, voici le véritable enjeu de l'écrivain symboliste.

\section{Les mystifications à la base de l'intrigue}

Loin de se limiter au pseudonyme de l'auteur et à ses ruses génériques, le principe de la mystification pénètre également à l'intérieur des romans, constituant parfois la base même de leurs intrigues. Mettons de côté À rebours (1884) de Huysmans, un «roman de l'artifice, du faux-vrai et du vrai-faux-vrai » (BERTRAND, 1996 : 22) ou Bruges-la-Morte de Rodenbach, ce «roman du reflet » (BERTRAND, 1996:33), et concentrons-nous sur des textes symbolistes moins connus qui érigent la mystification au rang d'une catégorie poétique :

Le roman Très russe (1886) de Jean Lorrain raconte l'histoire d'un écrivain appelé Mauriat, désireux de reconquérir la riche et mystérieuse $\mathrm{M}^{\mathrm{me}}$ Livitinof qu'il avait jadis possédée à Florence, mais qui se refuse désormais à lui. Le héros rivalise avec un autre prétendant, un certain Jean de Beaufrilan (vaguement inspiré de Guy de Maupassant), qu'il soupçonne d'être l'amant actuel de la belle courtisane. Un soir, arme à la main, Mauriat surprend sa bien-aimée attendant Beaufrilan à la terrasse de sa villa. Le dandy arrive, mais grâce à un stratagème concocté par $\mathrm{M}^{\text {me }}$ Livitinof, il finit par coucher sans le savoir avec sa servante Nitika. Mauriat et $\mathrm{M}^{\text {me }}$ Livitinof peuvent ainsi passer toute la nuit ensemble à se promener et à lire des vers. À la fin, la femme apprend à son prétendant qu'elle « a toujours eu des Nitikas », même à Florence. Donc, ni Beaufrilan ni Mauriat ni aucun autre admirateur n'a jamais réellement couché avec elle. Non seulement l'héroïne caricature ici le cliché traditionnel de la femme fatale et inaccessible qui castre ses prétendants, mais elle personnalise également une sorte d'intrigue moderne, vide, dépsychologisée, tournant sur elle-même. De la même manière que Mauriat n'aura jamais la belle $\mathrm{M}^{\text {me }}$ Livitinof, l'écrivain décadent tournera en vain devant une page blanche qui se refusera à son écriture.

Un schéma similaire sera utilisé par Remy de Gourmont dans Sixtine (1890) où Hubert d'Entragues diffère sans cesse le moment de conquérir la belle Sixtine 
Magne, au point de se la voir ravir par un étranger qu'il lui avait lui-même présenté. Tout comme l'héroïne éponyme, le roman semble s'escamoter à mesure qu'il se construit, allant de digression en digression et jouant sur l'alternance de formes diverses : notes de voyage, contes, poèmes, dissertations, énumérations, jeux de miroir.

Le sommet de ce genre de «roman célibataire» serait probablement Les Lauriers sont coupés (1887) d'Édouard Dujardin. Le texte met en scène Daniel Prince, un jeune homme médiocre qui, six heures durant et la plupart du temps dans une solitude totale, se prépare à une rencontre amoureuse qui se soldera par un échec cuisant. La lecture actuelle des Lauriers, à savoir leur interprétation comme un texte psychologique qui préfigure le «stream of consciousness » joycien, cache dans une large mesure l'aspect ironique voire comique du texte. En fait, Daniel Prince est une sorte de Candide à l'envers qui marche naïvement vers son Eldorado amoureux, sans jamais comprendre à quel point il est dupé, exploité, ridiculisé par sa Léa. Le narrateur ne manque pas une seule occasion de souligner l'écart existant entre la vie réelle d'une part et les idéaux du jeune dandy d'autre part. Les six heures de simulations et dissimilations n'aboutissent finalement sur aucune prise de conscience et le héros, tout aussi dupe qu'au début, remet le prochain rendez-vous «à mercredi, trois heures » pour recommencer le jeu du chat et de la souris.

Une très belle scène de mystification couronne également l'Ève future (1886) de Villiers de l'Isle-Adam: Le protagoniste Lord Ewald tombe désespérément amoureux d'Alicia Clary, une actrice très belle, mais malheureusement sotte et petite-bourgeoise. Au cri désespéré de l'amant malheureux «Ah! qui m'ôtera cette âme de ce corps! » (VILLIERS DE L'ISLEADAM, 1986 : 814) répond Thomas Alva Edison. Pour guérir son ami de l'atroce souffrance d'aimer une créature qu'il admire et méprise à la fois, l'ingénieur américain fabrique de toutes pièces une « andréïde » qui a l'apparence physique de la belle Alicia, mais qui lui est infiniment supérieure sur les plans intellectuel et moral.

La toute première rencontre entre Ewald et Hadaly, la femme artificielle enfin achevée, est savamment orchestrée par le scientifique américain : Dans le livre VI, chapitre IV du roman, au crépuscule d'une journée d'éclipse, le jeune aristocrate se promène dans un parc avec Alicia. Charmé par sa beauté, ému par l'intérêt inhabituel qu'elle semble manifester pour ses tourments, Ewald finit par déclarer son amour à la jeune femme et décide de renoncer définitivement à sa folle expérience avec l'andréïde :

«Ô mon amour! », murmurait-il, presque éperdu. [...] Étais-je donc insensé ? Je rêvais le sacrilège... d'un jouet - dont l'aspect seul m'eût fait sourire, j'en suis sûr ! - d'une absurde poupée insensible ! Comme si, devant une jeune femme aussi solitairement belle comme toi, ne s'évanouissaient pas toutes ces démences d'électricité, de pressions hydrauliques et de cylindres vivants ! Vraiment, je remercierai tout à l'heure Edison, et sans autre curiosité. [...]

En même temps, Miss Alicia Clary se leva - et, appuyant sur les épaules du jeune homme ses pâles mains chargées de bagues étincelantes, elle lui dit mélancoliquement, - mais de cette voix inoubliable et surnaturelle qu'il avait une fois entendue : «Ami, ne me reconnais-tu pas ? Je suis Hadaly. » (VILlieRs De L'ISLE-AdAM, 1986 : 982-983) 
Par un coup de théâtre spectaculaire, l'auteur renverse ici toutes les attentes du lecteur, car derrière une femme (enfin) aimante, compréhensive et capable de sentiments désintéressés se cache en fait l'andréïde fraîchement fabriquée par Edison. Autrement dit, le jeune lord a passé à son insu une sorte de test de Turing préparé par son ami. N'ayant pas été capable de distinguer une femme réelle d'une créature artificielle, il a résolument penché en faveur de la deuxième.

\section{L'esthétique de la simulation}

Comme nous pouvons le vérifier dans la plupart des romans symbolistes ou décadents, le principe de la mystification ne représente jamais un simple détail, un divertissement, une débanalisation de l'intrigue. Les nombreuses simulations et dissimulations fonctionnent soit comme des métaphores de l'écriture (impossibilité pour le sujet de se raconter sincèrement, angoisse de la page blanche, crise des procédés du roman réaliste, laboratoire de nouvelles formes romanesques pour le vingtième siècle, idée du work in progress contre la notion de l'œuvre achevée), soit comme des interrogations philosophiques vaguement schopenhaueresques : Qu'est-ce que le monde au juste ? Existe-t-il réellement en dehors de ma volonté et ma représentation ? Qu'en est-il du sujet pensant et sentant ? L'objet aimé préexiste-t-il à l'amour et à son traitement dans le texte ? Peut-on encore parler de «nature », ou bien vivons-nous depuis des siècles dans le monopole du culturel, de l'artificiel? La sincérité est-elle pensable voire souhaitable en société ? Dans quelle mesure une femme peut-elle suivre ses propres désirs? Le réel (à condition qu'il existe, ce qui devient de moins en moins sûr) est-il préférable à l'art et au rêve, le monde environnant peut-il recéler les richesses du monde intérieur ? Bref, existe-t-il autre chose que moi ? Et ce moi, ne s'éparpille-t-il pas en des milliers de masques contradictoires, constamment menacé par des contraintes sociales d'une part et remis en cause par les profondeurs de l'inconscient d'autre part.

Ce n'est pas un hasard si le principal document de l'époque qui traite des écrivains symbolico-décadents s'appelle Le Livre des masques (1896, 1898 pour le second tome) et si le livre synthétique de Gustave Le Rouge Verlainiens et décadents paraît dans une collection intitulée "Masques et idées » (1928). Une époque qui refuse l'homogénéité du sujet, la linéarité de l'intrigue romanesque, la logique positiviste ainsi que la mimésis traditionnelle du réalisme ne peut plus, à l'instar de Nietzsche, croire que «la vérité demeure vérité, lorsqu'on lui arrache le voile...» (NIETZSCHE, 1908 : 100). En effet, comme Anatole France le rappelle dans La Vie en fleur, "qui dit art dit arrangement, dissimulation, mensonge » (FRANCE, 1987 : 1174). En cette fin de siècle, les mouvements symboliste et décadent prônent, plus encore que leurs prédécesseurs historiques, l'artifice, la subversion, la mystification comme principes fondamentaux de leur poétique.

Cette vérité «objective », dont le positivisme se réclamait jadis comme de sa valeur suprême, semble aux nouvelles générations fin-de-siècle bien difficilement compatible avec la complexité du moi et la rapidité de la vie moderne, bien éloignée des secrets désirs et aspirations humaines : 
Considérez, monsieur, que la vérité a sur le mensonge des caractères d'infériorité qui la condamnent à disparaître. [...] Le mensonge étant multiple, elle a contre elle le nombre. Ce n'est pas son seul défaut. Elle est inerte. Elle n'est pas susceptible de modification; elle ne se prête pas aux combinaisons qui pourraient la faire entrer aisément dans l'intelligence ou dans les passions des hommes. Le mensonge, au contraire, a des ressources merveilleuses. Il est ductile, il est plastique. Et, de plus (ne craignons point de le dire) il est naturel et moral. Il est naturel comme le produit ordinaire du mécanisme des sens, source et réservoir d'illusions ; il est moral en ce qu'il s'accorde avec les habitudes des hommes qui, vivant en commun, ont fondé leur idée du bien et du mal, leurs lois divines et humaines, sur les interprétations les plus anciennes, les plus saintes, les plus absurdes, les plus augustes, les plus barbares et les plus fausses des phénomènes naturels. Le mensonge est le principe de toute vertu et toute beauté chez les hommes. (FRANCE, $1991: 81$ )

Bref, loin de se résumer à une simple provocation vis-à-vis des naturalistes dont le nouveau mouvement entend se démarquer, bien sûr, avec une certaine violence, l'éloge de la mystification qu'entreprennent les symbolistes fonctionne comme une sorte de préface gnoséologique : Les masques, les fards, les voiles, les artifices sont des métaphores indispensables pour s'attaquer à une « vérité » trop évidente, lisse et simplificatrice des esprits positivistes, triomphants, de plus en plus imbus d'eux-mêmes.

Le port du masque renvoie, au contraire, à un monde où les sujets se disloquent, les identités se brouillent, les certitudes anciennes volent en éclats. Tandis que les positivistes pratiquaient l'expérimentation scientifique pour «s'approcher de plus en plus de la vérité » (BERNARD, 1966:41) - qui semblait les attendre, sagement assise dans un coin du laboratoire -, les symbolistes, plus sceptiques quant aux dissections et autres interventions chirurgicales, veulent éveiller leurs lecteurs à l' « extrême conscience » (MICHELET-JACQUOD, 2008), une faculté quasi divinatoire qui se situe bien au-delà de la vie empirique. Et si l'homme/la femme ne relevait pas de la pure physiologie ? Si la réalité n'était pas aussi facilement prévisible, mesurable et calculable ? Si le déterminisme n'était pas une mécanique bien huilée, mais au contraire un jeu de forces obscures que Nietzsche et Freud, ces deux maîtres à penser fin-de-siècle, commencent à peine à discerner?

\section{Conclusion}

Vus d'aujourd'hui, les masques symbolistes préfigurent beaucoup de choses, de la psychanalyse à l'effet papillon, de la science-fiction à la cybernétique, des théories racistes au féminisme, du Nouveau roman à l'écriture postmoderne. Ils sont peut-être l'une des meilleures introductions à la complexité du vingtième siècle et à notre monde d'aujourd'hui. Sans tenir compte du fait que, sur un plan plus strictement littéraire, ils théorisent déjà ce qu'Aragon appellera plus tard le principe du «mentir-vrai »: Par un paradoxe magique, la fiction, l'œuvre d'art est plus à même de révéler la vérité profonde d'une époque, d'un être humain, qu'une étude historique, biologique, psychologique, anthropologique ou documentaire. (DOTAL, $2008: 152$ ) 
En effet, les véritables créateurs se servent de la vie comme d'une matière brute qu'il s'agit de recréer, remodeler, réinventer. Comme Oscar Wilde le résume :

[...] la Nature extérieure imite l'Art. Les seuls effets qu'elle puisse nous montrer sont ceux que nous avons déjà vus en poésie ou en peinture. C'est le secret du charme de la Nature aussi bien que l'explication de sa faiblesse. La révélation finale est que le Mensonge, le récit de belles choses fausses, est le but même de l'Art. (WILDE, 1998 : 72)

\section{BIBLIOGRAPHIE}

BAUDELAIRE Charles (1868), Le public moderne et la photographie, Curiosités esthétiques, Paris, Michel Lévy Frères, p. 254-263.

BAUDELAIRE Charles (1939), Puisque réalisme il y a, Euvres complètes de Charles Baudelaire, t. I, Paris, Louis Conard, p. 296-299.

BERNARD Claude (1966), Introduction à la médecine expérimentale, Paris, Garnier Flammarion.

BertRAND Jean-Pierre; BIRON Michel; DubOIS Jacques; PAQUE Jeannine (1996), Le roman célibataire. D’À rebours à Paludes, Paris, Corti.

CARAION Marta (2003), Pour fixer la trace. Photographie, littérature et voyage au milieu du XIX siècle, Genève, Droz.

Dotal Sabine (2008), Le mentir-vrai, La Vérité, $\mathrm{n}^{0} 34$, Société française de Gestalt, p. 152-162.

DUJARDIN Édouard (2001), Les Lauriers sont coupés, Paris, Flammarion.

FRANCE Anatole (1987), Euvres, tome II, Paris, Gallimard, «Bibliothèque de la Pléiade ».

FrANCE Anatole (1991), CEuvres, tome III, Paris, Gallimard, «Bibliothèque de la Pléiade ».

Gourmont Remy de (1898), Le Deuxième Livre des Masques, Paris, Mercure de France.

GOURMONT Remy de (1896), Le Livre des masques, Paris, Mercure de France.

Gourmont Remy de (1890), Sixtine. Roman de la vie cérébrale, Paris, Albert Savine.

HuYSMANS Joris-Karl (1884), À rebours, Paris, G. Charpentier.

IBELS André (1898), Enquête sur le roman illustré par la photographie, Mercure de France, janvier 1898, p. 97-115.

LORRAIN Jean (2008), Très russe, Paris, Julia H.

MICHELET JACQUOD Valérie (2008), Le Roman symboliste : un art de l'extrême conscience : Edouard Dujardin, André Gide, Remy de Gourmont, Marcel Schwob, Genève, Droz.

NIETZSCHE Friedrich (1908), Nietzsche contre Wagner. Pièces justifcatives d'un psychologue, traduit par Henri Albert, Paris, Mercure de France.

RACHILDE (1977), Monsieur Vénus, Paris, Flammarion.

RENARD Jules (1990), Journal, Paris, Laffont « Bouquins ».

RoDENBACH Georges (1998), Bruges-la-Morte, Paris, Flammarion. 
VILliERS DE L'ISLE-ADAM (1986), Cuvres complètes, tome I, Paris, Gallimard, « Bibliothèque de la Pléiade ».

WILDE Oscar (1998), Le Déclin du mensonge, Paris, Allia. 